\title{
IDENTIFYING USABILITY ISSUES WITH AN ERP IMPLEMENTATION
}

\author{
Heikki Topi, Wendy Lucas, Tamara Babaian \\ Bentley College, Computer Information Systems Department, Waltham, MA, 02452, USA \\ Email: htopi@bentley.edu,wlucas@bentley.edu,tbabaian@bentley.edu
}

Keywords: usability, ERP, collaboration, enterprise system, user

\begin{abstract}
Enterprise Resource Planning (ERP) systems hold great promise for integrating business processes and have proven their worth in a variety of organizations. Yet the gains that they have enabled in terms of increased productivity and cost savings are often achieved in the face of daunting usability problems. While one frequently hears anecdotes about the difficulties involved in using ERP systems, there is little documentation of the types of problems typically faced by users. The purpose of this study is to begin addressing this gap by categorizing and describing the usability issues encountered by one division of a Fortune 500 company in the first years of its large-scale ERP implementation. This study also demonstrates the promise of using collaboration theory to evaluate usability characteristics of existing systems and to design new systems. Given the impressive results already achieved by some corporations with these systems, imagine how much more would be possible if understanding how to use them weren't such an overwhelming task.
\end{abstract}

\section{INTRODUCTION}

Enterprise Resource Planning (ERP) systems and other large scale enterprise systems (such as Supply Chain Management, Customer Resource Management, etc.) are widely used in virtually all industries. In the 1990s, enterprise systems became a core part of the information systems architecture in a very large number of corporations (Davenport, 1998), even though many implementations have either failed entirely or have not reached the goals set for them (Davenport, 1998; Chen 2001; Scott \& Vessey, 2002). Not surprisingly, enterprise systems have been studied very actively, particularly by the information systems community (Esteves \& Pastor, 2001). Many of these studies have focused on the factors affecting the success of ERP implementations (Sarker \& Lee, 2003; Grossman \& Walsh, 2004; Siau, 2004).

One of the areas that has not been widely studied in either academic or practitioner literature is the usability of ERP systems and other large-scale enterprise systems. While usability problems may not lead directly to a large-scale failure, they can interfere with an individual's or workgroup's productivity, making it harder for users to achieve their goals as effectively or efficiently as is desirable. They may also make system acceptance a more difficult and lengthy process.

The long-term goal of our research is to develop a set of design principles, the application of which leads to improvements in the usability of ERP systems. While these principles would be applicable also to other large-scale systems, we are focusing on ERP systems in part because of their aforementioned widespread usage. Our contention is that improved ERP usability can be achieved by designing the system with collaboration as the model for usersystem interaction. The term collaboration should not be interpreted literally: a computer system does not have a free will and current technology is not capable of creating a system with the capacity to reason that is akin to a human collaborator. However, the metaphor of a system-partner, in which the goals of the user are understood and supported by the system, can be the framework for the design, leading to system interfaces and interactions that are more supportive of the user.

The user-system collaboration metaphor is especially relevant in the enterprise-wide context, where the system possesses a vast amount of organizational data and is responsible for automating a large number of business processes. One of the central principles of collaborative behaviour (Bratman, 1992) is the commitment to mutual 
support, which requires that when either party recognizes that the other one needs help to complete a subtask successfully, it will provide that help (assuming it is capable of doing so). Another central tenet is the commitment to joint activity, which requires that both parties recognize and commit to that activity. To make this commitment, the parties must be aware of the context surrounding their collaboration. A third principle is that of mutual responsiveness, in which each party adjusts its behaviour based on the behaviour of the other and guided by commitment to the joint activity.

In this paper, we report on the findings of our field study on an ERP system in one division of a Fortune 500 company. Gaining insight into the problems that users experience with existing ERP systems and understanding the tasks that those systems are intended to support are critical first steps in working toward improved system usability. Our findings confirm what can be surmised from the large bodies of anecdotal evidence; namely, that poor usability characteristics, such as unnecessarily complex tasks and inadequate system support in response to user errors, impact acceptance, usage, and the usefulness of ERP systems, even in successful implementations. These findings augment the results of a laboratory comparison evaluating the usability of a large number of ERP systems that was conducted by Forrester Research (Gilbert, 2003).

None of these problems are new: textbooks in systems analysis and design have warned against them since at least the 1980s. The novelty of our approach is in considering these flaws in light of collaboration theory. Our study proposes that the usability problems that greatly affect users' performance can be viewed as examples of noncollaborative behaviour by the system. Rather than "standing" idly or silently by when the data in its possession could help the user diagnose and fix a problem, such as missing information on an invoice, the system must work on the user's behalf as a collaborative partner.

The contributions made by this paper are as follows:

- It is the first attempt to focus on usability problems experienced by users of a large scale ERP implementation. This study was conducted in a division of a Fortune 500 company that had been using its ERP system for two and a half years, beginning with its launch in Summer 2001.

- We provide a classification of usability problems encountered by users and show that these flaws can be viewed as examples of non-collaborative behaviour by the system, thereby providing strong evidence in support of our contention that collaboration theory is a suitable conceptual framework for usability design and evaluation in the ERP context.

This work is part of a larger research project in which we are also developing prototypes whose design is driven by the central principles of collaborative behaviour (Babaian et al. 2004).

\section{RESEARCH METHODOLOGY \& CONTEXT}

The data used in this study was collected in ten indepth interviews with nine ERP users and one nonuser. The lengths of the interviews varied from 20 minutes to 90 minutes, both of which are outliers; the length of most interviews was about an hour. The interviews were conducted on the premises of the employer of our interviewees, i.e., the organization that had deployed the ERP system of interest. The interviews were semi-structured, starting with a set of predefined questions (available from the authors). The analysis of the data was performed on the interview transcripts, with the interviewers' notes from the interviews used as supporting evidence.

To maintain the confidentiality of the target organization, we do not describe it or the system at a detailed level. At a general level, the system is used to manage a challenging post purchase maintenance process for a large and complex engineering product. This ERP system is in use in five different locations worldwide, including our target business unit, where it has been used since 2001.

The respondents in the study were all employed by the same organization and, with the exception of the one non-user, were users of the same ERP system. The respondents represent a variety of organizational roles, ranging from shop floor operational workers to upper middle management. Even though the roles vary, all of the respondents need the system (or data generated from the system in the case of the non-user) to fulfil their daily tasks within the organization.

\section{ANALYSIS OF THE RESULTS}

The analysis of the interview data was based on the transcripts. The analysis process began with the creation of a classification structure derived from the observations made during the interview process and from an initial analysis of the transcripts. A research assistant then classified the entire interview material at a detailed level to identify usability problems; if necessary, new categories were added to the 
structure. The results of the research assistant's work served as the foundation from which the authors completed the final usability problem categorization presented below. The remainder of this section will discuss the identified usability problem categories and their significance from the perspective of collaboration theory.

A qualitative analysis of the interview transcripts led to the following categories of usability problems:

- Identification of and access to the correct functionality

- Transaction execution support

- $\quad$ System output limitations

- Support in error situations

- Terminology problems

- Overall system complexity

Following are descriptions and examples by category of the actual problems encountered by ERP system users.

\subsection{Identification of and access to the correct functionality}

Several users noted that finding specific functionality quickly within the system sometimes required an unreasonable amount of effort. A simple example of this is navigation; that is, finding a specific transaction. Navigation problems seemed to be typical, as illustrated by the following comments of users, at least one of whom has extensive knowledge and experience with multiple system modules:

But it's also very intensive, cumbersome; there's multiple transactions that unfortunately is [sic] not linked to basically a central menu area to walk you through. You have to kind of know what the transaction codes are and how to execute them.

Or maybe we have some menus, but presently it may take us four, five or six routes to get us to basically one screen. I don't always see the links.

The basic problem with navigation was that very little help was provided to the users by the system for determining the correct transaction screens and how they could be accessed most directly. The problem was most severe for those users who were not very familiar with the system and the screens needed to perform a specific business transaction.

A more significant problem was the users' difficulty in understanding and/or remembering which set of actions was necessary for completing a specific business process. It is very typical that the user of such a system has to perform a number of ERP transactions in order to complete one specific business process. Since the ERP system in our target organization was not designed to be aware of the business process the user was attempting to achieve, it was unable to guide the user through it, as indicated by the following comment:

There's nothing that says okay, I'm here. Act on me now. There's nothing that exists. ... No, [there's a ] transaction for ' $A$ ', a transaction for ' $B$ ', and a transaction for ' $C$ ' and nothing links them. Someone has to manually do each one of those.

After a while, the users had memorized the most common sequences, but it took a significant amount of time to get to that point. In some cases, the organization decided that understanding the correct ordering of ERP transactions to implement a particular business process was important enough to document on laminated "cheat sheets," as noted in the following quote:

And even the training resulted in some sheets being made out with the laminated plastic, with walk through menus for [a specific employee type], what to process, why you would process it and things like that, that were very beneficial.

Throughout the interviews, the lack of system support for understanding the business processes that mapped to the ERP tasks was identified as one of the most significant problems. One respondent expressed the importance of understanding and focusing on the business processes, rather than the system transactions:

But I can't stress enough the fact that the transactions are the easy part; it's the business that's the important piece.

When evaluated in the context of collaboration theory, the users' difficulties with navigation and understanding the correct transaction sequence for particular business processes suggest that the system design did not properly capture the composition of many high-level business tasks for which the system is used. As a result, the system cannot guide a user through the steps of a composite business process, and the user was left with the burden of searching for and memorizing the correct sequence of operations. This indicates that the system was designed without commitment to joint activity: the system cannot be committed to the user's goals if it has not been designed to be aware of them. 


\subsection{Transaction execution problems}

Many of the usability problems that the users faced were related to the completion of system transactions. Users often found the transaction interfaces unduly complex. This was evidenced by the cheat sheets developed in-house and used by the employees even after the training and by the customized data entry forms created to compress a transaction into fewer screens, thereby eliminating a lot of optional entry items.

Some transaction level problems were quite well-known, such as the one noted by this user's question:

...why do I have to keep entering the same data over and over?

A thoughtful collaborator would recognize the need for this repetitive data and would automatically enter it, as is typically done by many modern systems. Another, very experienced user expressed the same problem in different words:

Well, I mean, we're so used to copying and pasting. ... In some cases, it [the ERP system] remembers and will carry over to some of the screens, but not in all cases.

One of basic strengths of a computer system is in automating mundane data entry tasks, yet the ERP system left this job to the user. The problems related to the entry of redundant data are well known (increased errors, inconsistency between system components, increased storage needs, etc.). Given that the repetition of field values between different screens is derived from the underlying business processes, the system should automatically provide the repeated data in subsequent screens rather than requiring the user to either reenter or copy and paste it.

These transaction level problems indicate that the system lacked the capability of modifying its behavior based on the user's actions and was not supporting the user in situations where help was clearly needed.

\subsection{System output limitations}

One of the issues that came up repeatedly during the interviews was the difficulty in getting the desired output from the system. Some respondents expressed very strong and comprehensive opinions regarding specific reports that they needed to perform a specific task:
It's lousy, and it doesn't tell you anything; it doesn't give you the information you need, and it just makes you[r] work more difficult - I found it not easy to use, not the right information, didn't update correctly, didn't have a lot of flexibility.

This employee was not against the use of the system itself, but was, rather, an active user who was simply highly frustrated with the fact that he/she was not able to get the output that he/she knew was available through the system.

In many cases, unfulfilled reporting needs led to the downloading of a report or a set of raw data to an outside application (such as a Microsoft Excel ${ }^{\circledR}$ spreadsheet) in order to get the desired outcome.

And unless I export that down into [an] Excel file or something, the system [is not] capable of compressing that [data], to minimize it, reduce it.

Even experienced users felt that it would be highly useful to have an external expert helping with data output tasks. They accepted this as a given limitation that was related to the nature of this specific ERP and its data warehousing solution.

What you need is a couple of experts ... - hired ... we need to go out and we need to get a [data warehousing] expert to be able to sit there and listen to what we want, and then to be able to dig that information out of [the ERP system] and put it together and report that. That's the nature, I think, of [the ERP system].

The same user believed that using query tools to retrieve data from the system was excruciatingly difficult.

It's just that you need to be a brain surgeon to actually go out and ...... produce your own (queries).

The difficulties the users faced when attempting to retrieve the desired system outputs demonstrate that the system did not provide adequate support in helping users access the information they needed in a usable format.

\subsection{Support in error situations}

Some of the most significant and most commonly mentioned difficulties with using the system were caused by insufficient or misleading error messages. For example, some of the messages were too general to provide any useful information to the users: 
You've got to go see somebody about how come it's red. But that's after your transaction is completed. ... It just says transaction failed or something like that.

Many of the interviewees described a specific error message that appeared in a large number of different situations, causing a great deal of confusion and resulting in a lot of wasted time spent trying to determine the actual cause of the problem. Both examples below refer to this same error message:

Basically what [this error message] does is it gives you a generic error and you have to do the research to try to figure it out.

[This error message is] what I like to term the system throwing up its hands and saying "I don't know."

The users eventually identified several conditions that caused this particular error. Had the system reported the possible causes to the user, it would have saved them hours of confusion.

At times, the system failed to clearly communicate the type of error:

We have a screen where we try and return parts to the warehouse and if that's already been done, the transaction has already been done, it says '[ $a$ particularly cryptic error message]' on the bottom of the screen. What [expletive] does that mean to anybody?

Other times, important errors were ignored by the system, leading to potentially serious consequences:

We had the customized front-end and we had to hit two buttons to execute a transaction. But the system will allow you just to hit one. So the guys would hit one and everything would be green, hey, I must be okay, but they never created the other requirements that were necessary to [complete the transaction].

Effective communication between the parties is a prerequisite for the success of a collaborative effort. Clear, well-defined error messages and guidance concerning possible actions for the user to take would be some of the simplest ways for the system to provide adequate support.

\subsection{Terminology problems}

Another set of problems that affected the users' ability to make effective use of the ERP system arose from the fact that the system's terminology was different from users'.

The 'Help' is worthless because it's definitely programmer's language based. So having the 'Help' customized for business processes would be [an] important piece.

In some cases, specific remedies were implemented:

I put together a glossary of how the vocabulary changed from pre-[ERP] to post, because people didn't understand the terms.

Another user discussed the same situation with more colorful language, clearly indicating that the transition between the pre-ERP and post-ERP vocabularies was not an easy one

Well, it was like the spaceship had landed, and these outer space creatures [trainers] got off, and started talking to us about how we were going to do our job, because nobody understood what they were saying. Now, they're talking about notifications, material numbers, document control, material masters -- you know, that wasn't in any of our language.

The extent of the discrepancy between the two vocabularies was surprising:

So, we had to list down the side of everything that [ERP] brought in, okay? And then we checked off which ones were identical to the nomenclature that we had in legacy. Well, there were no checkmarks.

One of the respondents recognized that the terminology issue is not easy to solve - every company, after all, uses their own vocabulary. Recognition of the universality of this problem does not, however, solve it. For every organization, the vocabulary used for core business terms is an essential part of the communication toolkit between the members of the organization, and it does not change overnight.

These terminology-related problems indicate that the communication between collaborating parties was clearly impaired by the lack of a common vocabulary. Thus, the collaborative principle of mutual responsiveness was violated. 


\subsection{Overall system complexity}

Another negative characteristic of the ERP system that was commonly mentioned throughout the interview process was its overall complexity, as demonstrated by the following statement:

It's a very intimidating system. Some people are very intimidated.

and by this, more colorful, example:

Th[is] guy could do it [assemble a very complex engineering artifact] in his sleep. But tell him to get a ERP GUI, logon password and explain to him this is how [to execute a specific transaction using ERP] and this is what's going wrong and why you guys are doing this. Oh my god, he was like a deer; it was like he got so upset because it was so out of his kingdom, so out of his normal -he shutdown on me. He actually shut down on me.

A commonly expressed perception was that this particular ERP system was a very complex one to understand and use for a large portion of the users. While this perception might have been partially based on those users' computer anxiety, it was clear that general system characteristics at least contributed to their perplexity. Strong user perceptions of system complexity again suggest that the principles of mutual responsiveness and commitment to mutual support have been violated.

\section{CONCLUSION}

This field study highlights many of the issues users face when dealing with a large-scale ERP system. While the eventual outcome in this case was a success, the problems identified by the users clearly impacted the amount of time it took them to learn how to use the system, the number of errors resulting from a lack of understanding about the steps required to complete a process, and the level of frustration felt by the users due to ill formed error messages, unclear instructions, and the overall lack of system helpfulness. Identifying factors affecting the users' ability to make the most effective use of ERP systems is essential to understanding how the design of these systems can be improved. The potential impacts of enhancing usability are significant; less frustrated users with a clearer understanding of system usage will save organizations time and money through lower training costs, faster ramp-up times, and more complete usage of the system for all the tasks it was meant to handle.

In addition to identifying and categorizing usability problems of a large ERP system, this study also demonstrates the promise of using collaboration theory to evaluate usability characteristics of existing systems and to design new systems. All identified usability problem categories were explicit violations of at least one of the principles of collaboration theory.

Future work must focus on identifying the relative importance of each of the problem categories in terms of its impact on the organization, followed by the development of means for addressing these issues based on the guiding design principles derived from collaboration theory.

\section{REFERENCES}

Babaian, T., Lucas, W. \& Topi, H. 2004. Collaborating to Improve ERP Usability. In Proceedings of ICEIS'04

Bratman, M. E. 1992. Shared cooperative activity. The Philosophical Review, 101(2), pp. 327-341.

Chen, I.J. 2001. Planning for ERP systems: Analysis and future trend. Business Process Management Journal, 7(5), pp. 374-387.

Davenport, T.H. 1998. Putting the enterprise back into the enterprise system. Harvard Business Review, 76(4), 121-132.

Esteves, J. \& Pastor, J. 2001. Enterprise resources planning research: An annotated bibliography. Communications of the AIS, 7 (8), 1-52

Gilbert, A. 2003. Business apps get bad marks in usability. CNET News.com, http://news.com.com/ 2100-1017980648.html (current 11/7/2004).

Grosz, B. J. 1996. Collaborative systems. AI Magazine, 17(2), pp. 67-85.

Grossman, T. \& Walsh, J. 2004. Avoiding the Pitfalls of ERP System Implementation. Information Systems Management, 21(2), p. 38-42.

Sarker, S., \& Lee, A.S. 2003. Using a case study to test the role of three key social enablers in ERP implementation. Information \& Management, 40(8), 813-829

Scott, J.E., \& Vessey, I. 2002. Managing Risks in Enterprise Systems Implementations. Communications of the ACM, 45(4), 74-81.

Siau, K. 2004. Enterprise resource planning (ERP) implementation methodologies. Journal of Database Management, 15(1), 1-6 